\title{
Genetic polymorphisms of CCND1 and PTEN in progression of esophageal squamous carcinoma
}

\author{
Y. Jang', S.A. Lu' ${ }^{2}$, Z.P. Chen ${ }^{3}$, J. Ma ${ }^{3}$, C.Q. Xu ${ }^{3}$, C.Z. Zhang ${ }^{3}$ \\ and J.J. Wang ${ }^{4}$ \\ ${ }^{1}$ Medical Department, People's Hospital of Lanshan District, \\ Linyi, Shandong, China \\ ${ }^{2}$ Surgical Department, Jinan Central Hospital, Jinan, \\ Shandong, China \\ ${ }^{3}$ Department of Gastroenterology, Shandong Provincial Qianfoshan Hospital, \\ Jinan, Shandong, China \\ ${ }^{4}$ Clinical Laboratory, Shandong Provincial Qianfoshan Hospital, \\ Jinan, Shandong, China \\ Corresponding author: J.J.Wang \\ E-mail: wangjj0531@163.com
}

Genet. Mol. Res. 12 (4): 6685-6691 (2013)

Received February 4, 2013

Accepted October 6, 2013

Published December 13, 2013

DOI http://dx.doi.org/10.4238/2013.December.13.2

\begin{abstract}
Cyclin D1 (CCND1) plays a significant role in G1-S transition of cell cycle, and phosphatase and a tensin homologue (PTEN) negatively regulate cell cycle through phosphatidylinositol 3-kinase (PI3K)/AKT signaling. CCND1 and PTEN genetic polymorphisms might induce susceptibility to the occurrence of esophageal squamous cell carcinoma (ESCC). Three hundred and four ESCC patients and 413 healthy controls from Anyang, China, were enrolled in this study. All genotyping at CCND1 (807 G/A) and PTEN (rs701848 T/C and rs $2735343 \mathrm{C} / \mathrm{G}$ ) were identified by polymerase chain reaction-restriction fragment length polymorphism (PCR-RFLP) assay. Unconditional logistic regression model was used to analyze the correlation between the polymorphisms and the susceptibility to develop ESCC. Statistically significant differences were observed between cases and controls in
\end{abstract}


distribution of genotypes or alleles at PTEN rs $701848 \mathrm{~T} / \mathrm{C}$ and rs2735343 $\mathrm{C} / \mathrm{G}$, with either haplotype TG or CG possessing notably higher proportion in cases than in the controls. However, such difference could not be found in the distribution of the polymorphisms at CCND1 $807 \mathrm{G} / \mathrm{A}$. In summary, the polymorphisms of PTEN rs701848 T/C and rs2735343 $\mathrm{C} / \mathrm{G}$ might represent crucial modifying factors for development of ESCC.

Key words: Polymorphism; Esophageal squamous cell carcinoma; CCND1; PTEN

\section{INTRODUCTION}

Cyclin D1 (CCND1) is a key regulatory factor in cell cycle, induced by mitogenic signals to transition cells from the quiescent G1 phase to the proliferative S phase. High activity of CCND1 leads to premature cell passage through the G1-S transition, resulting in propagation of unrepaired DNA damage and accumulation of genetic errors, and then leading to abnormal cell proliferation (Hall and Peters, 1996). Therefore, CCND1 has been recognized as a promising biological marker in predicting tumor behavior (Salesiotis and Cullen, 2000).

Phosphatase and tensin homologue (PTEN) encodes a dual specificity protein phosphatase, which negatively regulates phosphatidylinositol 3-kinase (PI3K)/AKT signaling, a pathway with an established role in promoting cell cycle progression. Expression of PTEN could inhibit cell cycle progression by G1 arrest, inhibit cell migration, and induce cell apoptosis (Furnari, et al., 1998; Davies, et al., 1999). Hence, loss of PTEN activity is a common feature of cancers.

Esophageal squamous cell carcinoma (ESCC) is one of the most common fatal cancers worldwide, with a poor survival rate and a dramatically rising incidence. Therefore, a better understanding of etiology of this malignancy is crucial for early detection and prevention. To this purpose, comprehensive analysis of genetic alterations in tumors and identification of genes involved in tumorigenesis have been major focus. It has been well believed that genetic polymorphisms can result in subtle structural alteration, with an influence on protein expression or function. Many studies have reported that some relevant polymorphisms of CCND1 or PTEN are associated with an increased risk of some types of cancers (Sun et al., 2001; Jia et al., 2008; Pabalan et al., 2008; Rizvi et al., 2011). In the current study, the polymorphisms of CCND1 (807 G/A) and PTEN (rs701848 T/C and rs2735343 C/G) were selected to assess the relationship with the susceptibility of ESCC in Chinese population.

\section{MATERIAL AND METHODS}

\section{Study population}

In this study, 304 ESCC patients were recruited from Anyang Cancer Hospital, Henan, China, and confirmed by histopathological analysis of endoscopic biopsies or surgical specimens, without chemotherapy or radiotherapy before surgery. The controls comprised 413 healthy blood donors, randomly selected from the same region, without any known history of cancers or autoimmune diseases. According to the principle of case-control study, the controls were selected to match to the cases by inhabitation region, age, and gender. Informed consent was obtained from each subject. 


\section{DNA extraction}

Genomic DNA was extracted from peripheral blood leukocytes of each subject by proteinase $\mathrm{K}$ digestion, phenol-chloroform extraction, and ethanol precipitation. Before PCR was performed, DNA samples were stored at $-20^{\circ} \mathrm{C}$.

\section{Genotyping}

Genotypes were determined by polymerase chain reaction-restriction fragment length polymorphism (PCR-RFLP) assay. Detailed information about primers, restriction enzymes, length of PCR and digest products was summarized in Table S1. The PCR amplification was performed in a $25-\mathrm{mL}$ reaction mixture, containing $100 \mathrm{ng}$ genomic DNA, $0.2 \mathrm{mM} \mathrm{dNTP}, 0.2$ $\mathrm{mM}$ of each primer, and 2.5 U Taq DNA polymerase. Reaction was carried out with an initial denaturation of $5 \mathrm{~min}$ at $94^{\circ} \mathrm{C}$, followed by 35 cycles of $30 \mathrm{~s}$ at $94^{\circ} \mathrm{C}, 30 \mathrm{~s}$ at respective annealing temperatures, and $30 \mathrm{~s}$ at $72^{\circ} \mathrm{C}$, and 10 min extension at $72^{\circ} \mathrm{C}$. Then PCR products were digested by respective restriction enzyme at $37^{\circ} \mathrm{C}$ for $8 \mathrm{~h}$. The digested products were used to analyze genotypes by electrophoresis on 3\% agarose gel and photography by the Gel-Doc 2000. PCR products of the identified genotypes at each polymorphism site of the $10 \%$ samples were further confirmed by direct sequencing of PCR products.

\section{Statistical analysis}

All analyses were carried out by the SPSS software (version 13.0 for Windows, Chicago, USA). For cases and controls, the distribution of age was analyzed by Mann-Whitney test and those of other demographic variables by chi-square test. Hardy-Weinberg equilibrium was tested by chi-square test for the observed genotype frequencies. PHASE software (version 1.0, University of Chicago, IL, USA) was applied to construct haplotypes on the basis of the identified polymorphisms and to estimate haplotype frequencies. Unconditional logistic regression model, adjusted by age, gender, smoking and drinking, was performed to calculate odds ratio (OR) and $95 \%$ confidence interval $(95 \% \mathrm{CI})$ to estimate possible effects of genotypes or haplotypes on ESCC. All tests were two-sided and $\mathrm{P}<0.05$ was considered to be statistically significant.

\section{RESULTS}

\section{Distribution of demographic characteristics}

As shown in Table 1, there were no significant differences between cases and controls with respect to age by Mann-Whitney test $(\mathrm{P}=0.88)$. Similarly, no significant differences in terms of gender, smoking or drinking were found by chi-square test $(\mathrm{P}=0.89, \mathrm{P}=0.59, \mathrm{P}=0.18$, respectively).

\section{Correlation between selected genetic polymorphisms and ESCC}

Table 2 displayed that the distribution of genotype frequencies at these sites among cases and controls was in Hardy-Weinberg equilibrium $(\mathrm{P}>0.05)$. For PTEN rs701848 T/C, cases possessed higher frequency of genotype $\mathrm{TC}$ or $\mathrm{CC}$ than controls $(\mathrm{P}<0.01, \mathrm{P}<0.01)$. This might 
indicate that genotype TC or CC carriers were more prone to develop ECSS $(\mathrm{OR}=1.89,95 \% \mathrm{CI}$ $=1.36-2.63 ; \mathrm{OR}=1.79,95 \% \mathrm{CI}=1.17-2.76$, respectively). Naturally, the proportion of allele $\mathrm{C}$ at rs701848 was notably increased in cases compared with controls $(\mathrm{P}<0.01)$, and individuals with allele $\mathrm{C}$ would be more probable to suffer from ESCC $(\mathrm{OR}=1.48,95 \% \mathrm{CI}=1.17-1.79)$. Similarly, increase in the frequencies of genotypes CG and GG at PTEN rs 2735343 was noted in cases compared with controls $(\mathrm{P}<0.01, \mathrm{P}<0.01)$. Both of the variant genotypes inferred about threefold increased risk of $\mathrm{ESCC}(\mathrm{OR}=2.58,95 \% \mathrm{CI}=1.74-3.82 ; \mathrm{OR}=3.59,95 \% \mathrm{CI}=2.34-5.50$, respectively). In addition, the variant allele $\mathrm{G}$ was overrepresented in cases $(60.36 \%$ vs $44.43 \%$, $\mathrm{P}$ $<0.01)$, and would increase individuals' susceptibility to $\mathrm{ESCC}(\mathrm{OR}=1.90,95 \% \mathrm{CI}=1.54-2.35)$. However, no significantly statistical differences between the two groups were observed in distribution of genotypes or alleles at CCND1 $807(\mathrm{P}=0.09, \mathrm{P}=0.31, \mathrm{P}=0.34$, respectively).

Table 1. Distribution of demographic characteristics.
\begin{tabular}{lccc}
\hline Variables & Cases $(\%, \mathrm{~N}=304)$ & Controls $(\%, \mathrm{~N}=413)$ & $\mathrm{P}$ \\
\hline Age (years) & $59.73 \pm 8.47$ & $59.63 \pm 8.46$ & $0.88^{*}$ \\
Gender & $168(55.26)$ & $226(54.72)$ & \\
$\quad$ Male & $136(44.74)$ & $187(45.28$ & $0.89^{* *}$ \\
$\quad$ Female & $112(36.84)$ & $123(29.78)$ & \\
Smoking & $192(63.16)$ & $290(70.22)$ & $0.59^{* *}$ \\
$\quad$ Yes & $88(28.95)$ & $101(24.46)$ & \\
$\quad$ No & $216(71.05)$ & $312(75.54)$ & $0.18^{* *}$ \\
Drinking & Yes & \\
No &
\end{tabular}

*Mann-Whitney U-test, ** chi-square test.

Table 2. Correlation between selected genetic polymorphisms and ESCC.

\begin{tabular}{|c|c|c|c|c|}
\hline Genotype & Cases $(\%, N=304)$ & Controls $(\%, N=413)$ & $\mathrm{P}^{*}$ & OR $(95 \% \mathrm{CI}) *$ \\
\hline \multicolumn{5}{|c|}{ CCND1 807 G/A } \\
\hline GG & $64(21.05)$ & $108(26.15)$ & - & 1.00 \\
\hline GA & $156(51.32)$ & $191(46.25)$ & 0.09 & $0.73(0.50-1.05)$ \\
\hline $\mathrm{AA}$ & $84(27.63)$ & $114(27.60)$ & 0.31 & $0.81(0.53-1.22)$ \\
\hline Allele G & $284(46.71)$ & $407(49.23)$ & - & 1.00 \\
\hline Allele A & $324(53.29)$ & $419(50.73)$ & 0.34 & $0.90(0.73-1.11)$ \\
\hline \multicolumn{5}{|c|}{ PTEN rs701848 T/C } \\
\hline TT & $91(29.93)$ & $183(44.31)$ & - & 1.00 \\
\hline $\mathrm{TC}$ & $155(50.96)$ & $165(39.95)$ & $<0.01$ & $1.89(1.36-2.63)$ \\
\hline $\mathrm{CC}$ & $58(19.08)$ & $65(15.73)$ & $<0.01$ & $1.79(1.17-2.76)$ \\
\hline Allele $\mathrm{T}$ & $337(55.43)$ & $531(64.29)$ & - & \\
\hline Allele C & $271(44.57)$ & $295(35.71)$ & $<0.01$ & $1.48(1.17-1.79)$ \\
\hline \multicolumn{5}{|c|}{ PTEN rs2735343 C/G } \\
\hline $\mathrm{CC}$ & $45(14.80)$ & $139(33.66)$ & - & 1.00 \\
\hline CG & $151(49.67)$ & $181(43.83)$ & $<0.01$ & $2.58(1.74-3.82)$ \\
\hline GG & $108(35.53)$ & $93(22.52)$ & $<0.01$ & $3.59(2.34-5.50)$ \\
\hline Allele C & $241(39.64)$ & $459(55.57)$ & - & 1.00 \\
\hline Allele G & $367(60.36)$ & $367(44.43)$ & $<0.01$ & $1.90(1.54-2.35)$ \\
\hline
\end{tabular}

*Logistic regression model, adjusted by age, gender, smoking, and drinking.

\section{Correlation between relevant haplotypes and ESCC}

After identifying genotypes, haplotypes were constructed by PHASE software, cover- 
ing the two polymorphism sites of PTEN. Each haplotype was subsequently selected to estimate the risk of ESCC by unconditional logistic regression model, as represented in Table 3. Compared with controls, the increase in the distribution frequency of haplotype TG (--/TG or $\mathrm{TG} / \mathrm{TG})$ was noted in cases $(26.32$ vs $18.40 \%, \mathrm{P}<0.01 ; 7.66$ s $4.36 \%, \mathrm{P}<0.01$, respectively), which indicated that individuals with haplotype TG (--/TG or TG/TG) were more inclined to $\operatorname{ESCC}(\mathrm{OR}=1.67,95 \% \mathrm{CI}=1.17-2.39$; $\mathrm{OR}=2.03,95 \% \mathrm{CI}=1.08-3.81$, respectively $)$. Similarly, haplotype CG (--/CG or CG/CG) exhibited higher frequencies in cases than controls (52.96\% vs $36.08 \%, \mathrm{P}<0.01 ; 14.80$ vs $12.11 \%, \mathrm{P}<0.01$, respectively), and individuals with this haplotype were observed more susceptible to develop ESCC $(\mathrm{OR}=2.36,95 \% \mathrm{CI}=1.71$ 3.26 ; $\mathrm{OR}=1.96,95 \% \mathrm{CI}=1.24-3.13$, respectively). Conversely, other haplotypes did not show such significant statistical differences $(\mathrm{P}=0.14, \mathrm{P}=0.78 ; \mathrm{P}=0.20, \mathrm{P}=0.08)$.

\begin{tabular}{|c|c|c|c|c|}
\hline Haplotype & Cases (\%) N = 304 & Controls (\%) $\mathrm{N}=413$ & $\mathrm{P}^{*}$ & OR $(95 \% \mathrm{CI})^{*}$ \\
\hline \multicolumn{5}{|l|}{ TC } \\
\hline$--/--$ & $88(28.95)$ & $97(23.49)$ & - & 1.00 \\
\hline$--/ \mathrm{TC}$ & $136(44.74)$ & $190(46.00)$ & 0.20 & $1.27(0.88-1.82)$ \\
\hline $\mathrm{TC} / \mathrm{TC}$ & $80(26.32)$ & $126(30.51)$ & 0.08 & $1.43(0.96-2.14)$ \\
\hline \multicolumn{5}{|l|}{$\mathrm{CC}$} \\
\hline$--/--* *$ & $273(89.80)$ & $382(92.49)$ & - & 1.00 \\
\hline$--/ \mathrm{CC}$ & $19(6.25)$ & $16(3.87)$ & 0.14 & $0.60(0.31-1.18)$ \\
\hline $\mathrm{CC} / \mathrm{CC}$ & $12(3.95)$ & $15(3.63)$ & 0.78 & $0.89(0.41-1.94)$ \\
\hline \multicolumn{5}{|l|}{ TG } \\
\hline$--/--$ & $201(66.12)$ & $319(77.24)$ & - & 1.00 \\
\hline$--/ \mathrm{TG}$ & $80(26.32)$ & $76(18.40)$ & $<0.01$ & $1.67(1.17-2.39)$ \\
\hline $\mathrm{TG} / \mathrm{TG}$ & $23(7.66)$ & $18(4.36)$ & 0.03 & $2.03(1.08-3.81)$ \\
\hline \multicolumn{5}{|l|}{ CG } \\
\hline$--/--$ & $98(32.24)$ & $214(51.82)$ & - & 1.00 \\
\hline$--/ C G$ & $161(52.96)$ & $149(36.08)$ & $<0.01$ & $2.36(1.71-3.26)$ \\
\hline $\mathrm{CG} / \mathrm{CG}$ & $45(14.80)$ & $50(12.11)$ & $<0.01$ & $1.96(1.24-3.13)$ \\
\hline
\end{tabular}

*Logistic regression model, adjusted by age, gender, smoking, and drinking. (--) = Any other haplotype, for example: --/TC indicates haplotype a in combination with any other haplotype.

\section{DISCUSSION}

Given the high morbidity and mortality, a better understanding of the genetic risk factors that underlie ESCC could lead to improved strategies for screening and prevention. Like most malignances, it is primarily a consequence of abnormal cell proliferation induced by uncontrolled cell cycle.

PTEN exerts its tumor suppressor function by acting as a negative regulator in the PI3K signaling pathway. It can inhibit cell growth, promote apoptosis, and regulate cell adhesion, migration, diffusion, and differentiation. Loss of PTEN activity has been identified in a number of human malignancies (Sun et al., 2001; Rizvi et al., 2011). Although rs701848 $\mathrm{T} / \mathrm{C}$ and $\mathrm{rs} 2735343 \mathrm{C} / \mathrm{G}$ are respectively located at intron and non-coding region, not able to change the encoded amino acids, they might influence genetic splicing, protein expression, regulation of cell cycle, and thus the risk of cancers. Particularly, rs701848 is located in 3'-UTR, which targeted by miRNA might alter the strength of miRNA binding, with consequence on regulation of target genes, thereby affecting the individuals' cancer risk (Brendle et al., 2008; Chin et al., 2008; Landi et al., 2008). In this study, the distributions of genotypes 
or alleles in cases, at both genetic sites of PTEN were statistically different from those in controls. Individuals with heterozygotes, variant homozygotes, or variant alleles at both sites (genotype TC, genotype CC or allele C at rs701848; genotype CG, genotype GG or allele $\mathrm{G}$ at rs 2735343 , respectively) were more prone to suffer from ESCC, in line with our previous studies and supposed mechanisms (Ma et al., 2012).

CCND1 is a key protein for the regulation of G1-S transition and considered an oncoprotein. The polymorphism of $807 \mathrm{G} / \mathrm{A}$ increases the frequency of alternate splicing, leading to an altered protein that does not contain the sequences involved in protein turnover and may have a longer half-life (Betticher et al., 1995). However, different studies indicate that the polymorphism might not be involved in the predisposition to ESCC (Yu et al., 2003; Liu et al., 2010), whereas some other show contrasting results (Izzo et al., 2005, 2007). Other genetic variations of cell cycle related proteins, such as retinoblastoma and $\mathrm{p} 21$, which interact with CCND1, were found. Thereby, the possibility that the polymorphism $807 \mathrm{G} / \mathrm{A}$ might confer susceptibility to ESCC via functional interaction with other genetic polymorphisms could not be excluded. Moreover, this discrepancy could be due to genetic differences or smaller population.

As genetic interactions are complex, many polymorphisms are silent without direct effects on the associated amino acids. However, the collective influence of several polymorphisms or haplotype may exert natural combinational or synergistic protection against diseases, including tumors. For the haplotypes constructed by PTEN, rs701848 T/C, and rs2735343 $\mathrm{C} / \mathrm{G}$, people with haplotype TC or CG would be more susceptible to ESCC, in line with the variant allele $\mathrm{G}$ at rs 2735343 contributing to the occurrence of ESCC, and indicating that this genetic polymorphism is more influent for ESCC than the other.

Our group has previously discussed the relationship between the polymorphism of PTEN and the susceptibility to ESCC in a much larger sample of Chinese population (Ma et al., 2012). In the present study, we further support the risk effect of the CCND1 polymorphism on ESCC in a relatively large population.

Thus, in this study, we report that the genetic polymorphisms of PTEN might contribute to the development of ESCC. In particular, the variant genotypes and alleles at rs 701848 or rs2735343 might be susceptible factors for ESCC, with haplotype TG or CG increasing individual tendency to ESCC. Additional studies and more functional experiments will be carried out to further validate the great value of the present results and their application in early diagnosis and treatments of ESCC.

\section{Supplementary material}

\section{REFERENCES}

Betticher DC, Thatcher N, Altermatt HJ, Hoban P, et al. (1995). Alternate splicing produces a novel cyclin D1 transcript. Oncogene 11: 1005-1011.

Brendle A, Lei H, Brandt A, Johansson R, et al. (2008). Polymorphisms in predicted microRNA-binding sites in integrin genes and breast cancer: ITGB4 as prognostic marker. Carcinogenesis 29: 1394-1399.

Chin LJ, Ratner E, Leng S, Zhai R, et al. (2008). A SNP in a let-7 microRNA complementary site in the KRAS 3' untranslated region increases non-small cell lung cancer risk. Cancer Res. 68: 8535-8540.

Davies MA, Koul D, Dhesi H, Berman R, et al. (1999). Regulation of Akt/PKB activity, cellular growth, and apoptosis in prostate carcinoma cells by MMAC/PTEN. Cancer Res. 59: 2551-2556.

Furnari FB, Huang HJ and Cavenee WK (1998). The phosphoinositol phosphatase activity of PTEN mediates a serum- 
sensitive G1 growth arrest in glioma cells. Cancer Res. 58: 5002-5008.

Hall M and Peters G (1996). Genetic alterations of cyclins, cyclin-dependent kinases, and Cdk inhibitors in human cancer. Adv. Cancer Res. 68: 67-108.

Izzo JG, Malhotra U, Wu TT, Ensor J, et al. (2005). Impact of cyclin D1 A870G polymorphism in esophageal adenocarcinoma tumorigenesis. Semin. Oncol. 32: S11-S15.

Izzo JG, Wu TT, Wu X, Ensor J, et al. (2007). Cyclin D1 guanine/adenine 870 polymorphism with altered protein expression is associated with genomic instability and aggressive clinical biology of esophageal adenocarcinoma. $J$. Clin. Oncol. 25: 698-707.

Jia A, Gong J, Li Y, Hao Z, et al. (2008). GG genotype of cyclin D1 G870A polymorphism is associated with non-cardiac gastric cancer in a high-risk region of China. Scand. J. Gastroenterol. 43: 1353-1359.

Landi D, Gemignani F, Naccarati A, Pardini B, et al. (2008). Polymorphisms within micro-RNA-binding sites and risk of sporadic colorectal cancer. Carcinogenesis 29: 579-584.

Liu G, Cescon DW, Zhai R, Zhou W, et al. (2010). p53 Arg72Pro, MDM2 T309G and CCND1 G870A polymorphisms are not associated with susceptibility to esophageal adenocarcinoma. Dis. Esophagus. 23: 36-39.

Ma J, Zhang J, Ning T, Chen Z, et al. (2012). Association of genetic polymorphisms in MDM2, PTEN and P53 with risk of esophageal squamous cell carcinoma. J. Hum. Genet. 57: 261-264.

Pabalan N, Bapat B, Sung L, Jarjanazi H, et al. (2008). Cyclin D1 Pro241Pro (CCND1-G870A) polymorphism is associated with increased cancer risk in human populations: a meta-analysis. Cancer Epidemiol. Biomark. Prev. 17: 2773-2781.

Rizvi MM, Alam MS, Ali A, Mehdi SJ, et al. (2011). Aberrant promoter methylation and inactivation of PTEN gene in cervical carcinoma from Indian population. J. Cancer Res. Clin. Oncol. 137: 1255-1262.

Salesiotis AN and Cullen KJ (2000). Molecular markers predictive of response and prognosis in the patient with advanced squamous cell carcinoma of the head and neck: evolution of a model beyond TNM staging. Curr. Opin. Oncol. 12: 229-239.

Sun H, Enomoto T, Fujita M, Wada H, et al. (2001). Mutational analysis of the PTEN gene in endometrial carcinoma and hyperplasia. Am. J. Clin. Pathol. 115: 32-38.

Yu C, Lu W, Tan W, Xing D, et al. (2003). Lack of association between CCND1 G870A polymorphism and risk of esophageal squamous cell carcinoma. Cancer Epidemiol. Biomark. Prev. 12: 176. 